\title{
PRODUCTS OF HUREWICZ SPACES IN THE LAVER MODEL
}

\author{
DUŠAN REPOVŠ AND LYUBOMYR ZDOMSKYY
}

\begin{abstract}
This article is devoted to the interplay between forcing with fusion and combinatorial covering properties. We illustrate this interplay by proving that in the Laver model for the consistency of the Borel's conjecture, the product of any two metrizable spaces with the Hurewicz property has the Menger property.
\end{abstract}

\section{INTRODUCTION}

A topological space $X$ has the Menger property (or, alternatively, is a Menger space) if for every sequence $\left\langle\mathcal{U}_{n}: n \in \omega\right\rangle$ of open covers of $X$ there exists a sequence $\left\langle\mathcal{V}_{n}: n \in \omega\right\rangle$ such that each $\mathcal{V}_{n}$ is a finite subfamily of $\mathcal{U}_{n}$ and the collection $\left\{\cup \mathcal{V}_{n}: n \in \omega\right\}$ is a cover of $X$. This property was introduced by Hurewicz, and the current name (the Menger property) is used because Hurewicz proved in [12] that for metrizable spaces his property is equivalent to one property of a base considered by Menger in [16]. If in the definition above we additionally require that $\left\{\cup \mathcal{V}_{n}: n \in \omega\right\}$ is a $\gamma$ cover of $X$ (this means that the set $\left\{n \in \omega: x \notin \cup \mathcal{V}_{n}\right\}$ is finite for each $x \in X)$, then we obtain the definition of the Hurewicz property introduced in [13]. Each $\sigma$-compact space is obviously a Hurewicz space, and Hurewicz spaces have the Menger property. Contrary to a conjecture of Hurewicz the class of metrizable spaces having the Hurewicz property appeared to be much wider than the class of $\sigma$-compact spaces [14, Theorem 5.1]. The properties of Menger and Hurewicz are classical examples of combinatorial covering properties of topological spaces which are nowadays also called selection principles. This is a growing area of general topology, see, e.g., [29]. For instance, Menger and Hurewicz spaces found applications in such areas as forcing [9], Ramsey theory in algebra [30], combinatorics of discrete subspaces [1], and Tukey relations between hyperspaces of compacts [10].

Even before the era of combinatorial covering properties, there was a lot of activity around the study of special sets of reals. These studies resolved many classical questions in general topology and measure theory. As a result, information about special sets of reals is included in standard topology textbooks, such as Kuratowski's Topology. The most influential survey on special sets of reals is, probably, Miller's chapter [18] in the Handbook of

2010 Mathematics Subject Classification. Primary: 03E35, 54D20. Secondary: 54C50, $03 \mathrm{E} 05$.

Key words and phrases. Menger space, Hurewicz space, semifilter, Laver forcing. 
Set-Theoretic Topology. The most recent monograph on this topic is written by Bukovsky, see [7]. It complements nicely the classical book [4] of Bartoszynski and Judah. This theory still finds interesting applications in general topology, see, e.g., [11] for the interplay between $\lambda$-sets and homogeneity.

The theory of combinatorial covering properties, which originated in $[14,24]$, can be thought of as a continuation to that of special sets of reals, with emphasis on the behaviour of their open or Borel covers. Some combinatorial covering properties including the Menger and Hurewicz ones are about 15 years older than Gödel's works on $L$ and 40 years older than the method of forcing, and they were introduced in the areas of topology where set-theoretic methods are quite rare even nowadays. E.g., the original idea behind the Menger property, as it is explicitly stated in the first paragraph of [16], was an application in dimension theory. However, since at least [15] it has become clear that the combinatorial covering properties are strongly influenced by axiomatics and hence can be studied with the help of forcing, see, e.g., [3, 8, 9, 19, 25] for the more recent works along these lines. There are equivalences among statements from disciplines with diverse origins (Ramsey theory, game theory, function spaces and convergence, topological groups, dimension theory, covering properties, combinatorial set theory, forcing, hyperspaces, filters, etc.) with combinatorial covering properties. Even though not all of these have found non-trivial applications so far (by translating into the other fields, via an equivalence, the results known for combinatorial covering properties), they are offering an alternative point of view onto the known properties and thus enhance their understanding. E.g., it is shown in [9] that a Mathias forcing associated to a filter $\mathcal{F}$ on $\omega$ does not add dominating reals iff $\mathcal{F}$ is Menger as a subspace of $2^{\omega}$, thus demonstrating that this property of filters is topological and in this way answering some questions for which it was unclear how the "standard" approaches in this area can be used.

One of the basic questions about a topological property is whether it is preserved by various kinds of products in certain classes of spaces. As usually, the preservation results may be divided into positive, asserting that properties under consideration are preserved by products (e.g., the classical Tychonoff theorem), and negative which are typically some constructions of spaces possessing certain property whose product fails to have it (e.g., the folklore fact that the Lindelöf property is not preserved even by squares, as witnessed by the Sorgenfrei line). In case of combinatorial covering properties we know that the strongest possible negative result is consistent: Under $\mathrm{CH}$ there exist $X, Y \subset \mathbb{R}$ which have the $\gamma$-space property with respect to countable Borel covers, whose product $X \times Y$ is not Menger, see [21, Theorem 3.2]. Thus the product of spaces with the strongest combinatorial covering property considered thus far might fail to have even the weakest one. This implies that no positive results for combinatorial covering properties can be obtained outright in ZFC. Unlike the vast majority of topological and combinatorial consequences under $\mathrm{CH}$, the latter one does 
not follow from any equality among cardinal characteristics of the continuum, see the discussion on [21, p. 2882]. However, there are many other negative results stating that under certain equality among cardinal characteristics (e.g., $\operatorname{cov}(\mathcal{N})=\operatorname{cof}(\mathcal{N}), \mathfrak{b}=\mathfrak{d}$, etc. $\left.{ }^{1}\right)$ there are spaces $X, Y \subset \mathbb{R}$ with some combinatorial covering property such that $X \times Y$ is not Menger, see, e.g., [2, 22, 27].

Regarding the positive results, until recently the most unclear situation was with the Hurewicz property and the weaker ones. This was the main motivation for this article. There are two reasons why a product of Hurewicz spaces $X, Y$ can fail to be Hurewicz/Menger. In the first place, $X \times Y$ may simply fail to be a Lindelöf space, i.e., it might have an open cover $\mathcal{U}$ without countable subcover. Then $X \times Y$ is not even a Menger space. This may indeed happen: in ZFC there are two normal spaces $X, Y$ with a covering property much stronger than the Hurewicz one such that $X \times Y$ does not have the Lindelöf property, see [28, Section 3]. However, the above situation becomes impossible if we restrict our attention to metrizable spaces. This second case, on which we concentrate in the sequel, turned out to be sensitive to the ambient set-theoretic universe: under $\mathrm{CH}$ there exists a Hurewicz space whose square is not Menger, see [14, Theorem 2.12]. The above result has been achieved by a transfinite construction of length $\omega_{1}$, using the combinatorics of the ideal of measure zero subsets of reals. This combinatorics turned out [27, Theorem 43] to require much weaker set-theoretic assumptions than $\mathrm{CH}$. In particular, under the Martin Axiom there are Hurewicz subspaces of the irrationals whose product is not Menger.

The following theorem, which is the main result of this article, shows that an additional assumption in the results from [14, 27] mentioned above is really needed. In addition, it implies that the affirmative answer to [14, Problem 2] is consistent, see [29, Section 2] for the discussion of this problem.

Theorem 1.1. In the Laver model for the consistency of the Borel's conjecture, the product of any two Hurewicz spaces has the Menger property provided that it is a Lindelöf space. In particular, the product of any two Hurewicz metrizable spaces has the Menger property.

This theorem seems to be the first "positive" consistency result related to the preservation by products of combinatorial covering properties weaker than the $\sigma$-compactness, in which no further restrictions ${ }^{2}$ on the spaces are assumed. The proof is based on the analysis of continuous maps and names for reals in the model of set theory constructed in [15]. The question whether the product of Hurewicz metrizable spaces is a Hurewicz space in this model remains open. It is worth mentioning here that in the Cohen model there

\footnotetext{
${ }^{1}$ We refer the reader to [5] for the definitions and basic properties of cardinal characteristics of the continuum which are mentioned but are not used in the proofs in this article.

${ }^{2}$ The requirement that the product must be Lindelöf is vacuous for metrizable spaces. Let us note that nowadays the study of combinatorial covering properties concentrates mainly on sets of reals.
} 
are Hurewicz subsets of $\mathbb{R}$ whose product has the Menger property but fails to have the Hurewicz one, see [21, Theorem 6.6].

As suggested in its formulation, the model we use in Theorem 1.1 was invented by Laver in order to prove that Borel's conjecture is consistent, the latter being the statement that every strong measure zero set is countable. A strong measure zero set is a subset $A$ of the real line with the following property: for every sequence $\left\langle\varepsilon_{n}: n \in \omega\right\rangle$ of positive reals there exists a sequence $\left\langle I_{n}: n \in \omega\right\rangle$ of intervals such that $\left|I_{n}\right|<\varepsilon_{n}$ for all $n$ and $A$ is covered by the $I_{n}$ 's. Here $\left|I_{n}\right|$ denotes the length of $I_{n}$. Obviously, every countable set is a strong measure zero set, and so is every union of countably many strong measure zero sets. Sierpiński proved in [26] that $\mathrm{CH}$ implies the existence of uncountable strong measure zero sets, i.e., the negation of Borel's conjecture. Combined with this Laver's result gave the independence of Borel's conjecture. This outstanding result was the first ${ }^{3}$ instance when a forcing, adding a real, was iterated with countable supports without collapsing cardinals. This work of Laver can be thought of as one of the motivations behind Baumgartner's axiom $A$ and later Shelah's theory of proper forcing.

The conclusion of Theorem 1.1 does not follow from Borel's conjecture: If we add $\omega_{2}$ many random reals over the Laver model then Borel's conjecture still holds by [4, Section 8.3.B] and we have $\operatorname{cov}(\mathcal{N})=\operatorname{cof}(\mathcal{N})$, and hence in this model there exists a Hurewicz set of reals whose square is not Menger, see [27]. Thus Borel's conjecture is consistent with the existence of a Hurewicz set of reals with nonMenger square.

Theorem 1.1 seems to be an instance of a more general phenomena, namely that proper posets with fusion affect the behavior of combinatorial covering properties. This happens because sets of reals with certain combinatorial covering properties are forced to have a rather clear structure, which suffices to prove positive preservation results. For instance, the core of the proof of Theorem 1.1 is that Hurewicz subspaces of the real line are concentrated in a sense around their "simpler" subspaces in the Laver model, see Lemma 2.2. As a consequence of corresponding structural results we have proved [33] that the Menger property is preserved by finite products in the Miller model constructed in [17], and there are only $\mathfrak{c}$ many Menger subspaces of $\mathbb{R}$ in the Sacks model constructed in [23], see [10].

We believe that the interplay between forcing with fusion and combinatorial covering properties has many more instances and it is worth considering whether there is some deep reason behind it.

We assume that the reader is familiar with the basics of forcing as well as with standard proper posets used in the set theory of reals.

\section{Proof of Theorem 1.1.}

We shall first introduce a notion crucial for the proof of Theorem 1.1.

Definition 2.1. A topological space $X$ is called weakly concentrated if for every collection $\mathrm{Q} \subset[X]^{\omega}$ which is cofinal with respect to inclusion, and

\footnotetext{
${ }^{3}$ According to our colleagues who worked in set theory already in the 1970s.
} 
for every function $R: \mathrm{Q} \rightarrow \mathcal{P}(X)$ assigning to each $Q \in \mathrm{Q}$ a $G_{\delta}$-set $R(Q)$ containing $Q$, there exists $\mathrm{Q}_{1} \in[\mathrm{Q}]^{\omega_{1}}$ such that $X \subset \bigcup_{Q \in \mathrm{Q}_{1}} R(Q)$.

The topology in $\mathcal{P}(\omega)$ is generated by the countable base $\mathcal{B}=\{[s, n]$ : $\left.s \in[\omega]^{<\omega}, n \in \omega\right\}$, where $[s, n]=\{x \subset \omega: x \cap n=s\}$. Thus any open subset $O$ of $\mathcal{P}(\omega)$ may be identified with $B_{O}=\left\{\langle s, n\rangle \in[\omega]^{<\omega} \times \omega:[s, n] \subset O\right\}$, and vice versa, any $B \subset[\omega]^{<\omega} \times \omega$ gives rise to an open $O_{B}=\bigcup_{\langle s, n\rangle \in B}[s, n]$. Note that $B \subset B_{O_{B}}$ for all $B$. By a code for an $F_{\sigma}$ subset $F$ of $\mathcal{P}(\omega)$ we mean a sequence $\vec{B}=\left\langle B_{n}: n \in \omega\right\rangle$ of subsets of $[\omega]^{<\omega} \times \omega$ such that $F=\mathcal{P}(\omega) \backslash \bigcap_{n \in \omega} O_{B_{n}}$. Obviously each $F_{\sigma}$ set $F \subset \mathcal{P}(\omega)$ has many codes in the sense of the definition above. For models $V \subsetneq V^{\prime}$ of ZFC and an $F_{\sigma}$-subset $F \in V^{\prime}$ of $\mathcal{P}(\omega)$ we say that $F$ is coded in $V$ if there exists a code for $F$ which is an element of $V$. Note that being coded in $V$ doesn't imply being a subset of $V: \mathcal{P}(\omega)$ has codes in $V$ (e.g., $\langle\langle\emptyset, 0\rangle: n \in \omega\rangle$ ) but it is not a subset of $V$ as long as there are new reals in $V^{\prime}$.

The consideration above also applies to other Polish spaces having a base which can be identified with some "simple" (e.g., constructive would suffice for our purposes) subset of $H(\omega)$, the family of all hereditarily finite sets. Among them are $\omega^{\omega}, \mathcal{P}(\omega) \times \omega^{\omega}$, etc. In particular, since every continuous function from an $F_{\sigma}$-subset $F$ of $\mathcal{P}(\omega)$ to $\omega^{\omega}$ is an $F_{\sigma}$-subset of $\mathcal{P}(\omega) \times \omega^{\omega}$, we may speak about such functions coded in $V$.

For a subset $X \in V^{\prime}$ of $\mathcal{P}(\omega)$ and an $F_{\sigma}$-subset $Y$ of $X$ we shall say that $Y$ is coded in $V$ if there exists an $F_{\sigma}$-subset $F$ of $\mathcal{P}(\omega)$ coded in $V$ such that $Y=X \cap F$. Similarly, for continuous functions: $f: Y \rightarrow \omega^{\omega}$ is coded in $V$ if there exists an $F_{\sigma}$-subset $F$ of $\mathcal{P}(\omega)$ such that $Y=X \cap F$, and a continuous $\tilde{f}: F \rightarrow \omega^{\omega}$ coded in $V$, such that $f=\tilde{f} \uparrow Y$.

The following lemma is the key part of the proof of Theorem 1.1. Its proof is reminiscent of that of [19, Theorem 3.2]. We will use the notation from [15] with only differences being that smaller conditions in a forcing poset are supposed to carry more information about the generic filter, and the ground model is denoted by $V$.

A subset $C$ of $\omega_{2}$ is called an $\omega_{1}-c l u b$ if it is unbounded and for every $\alpha \in \omega_{2}$ of cofinality $\omega_{1}$, if $C \cap \alpha$ is cofinal in $\alpha$ then $\alpha \in C$.

Lemma 2.2. In the Laver model every Hurewicz subspace of $\mathcal{P}(\omega)$ is weakly concentrated.

Proof. We work in $V\left[G_{\omega_{2}}\right]$, where $G_{\omega_{2}}$ is $\mathbb{P}_{\omega_{2}}$-generic and $\mathbb{P}_{\omega_{2}}$ is the iteration of length $\omega_{2}$ with countable supports of the Laver forcing, see [15] for details.

It is well known that a space $X \subset \mathcal{P}(\omega)$ is Hurewicz if and only if $f[X]$ is bounded with respect to $\leq^{*}$ for every continuous $f: X \rightarrow \omega^{\omega}$, see [14, Theorem 4.4] or [13]. Let us fix a Hurewicz space $X \subset \mathcal{P}(\omega)$. The Hurewicz property is preserved by $F_{\sigma}$-subspaces because it is obviously preserved by closed subspaces and countable unions. Therefore there exists an $\omega_{1}$-club $C \subset \omega_{2}$ such that for every $\alpha \in C$ and continuous $f: F \rightarrow \omega^{\omega}$ coded in $V\left[G_{\alpha}\right]$, where $F$ is an $F_{\sigma}$-subspace of $X$ coded in $V\left[G_{\alpha}\right]$, there exists $b \in \omega^{\omega} \cap V\left[G_{\alpha}\right]$ such that $f(x) \leq^{*} b$ for all $x \in F$. Indeed, since for every $\alpha<\omega_{2} \mathrm{CH}$ holds in $V\left[G_{\alpha}\right]$, there are at most $\omega_{1}$ many pairs $\langle F, f\rangle$ 
such that $F$ is an $F_{\sigma}$-subspace of $X$ coded in $V\left[G_{\alpha}\right]$ and $f: F \rightarrow \omega^{\omega}$ is a continuous function coded in $V\left[G_{\alpha}\right]$. For every such pair find $\gamma_{F, f}<\omega_{2}$ and $b_{F, f} \in \omega^{\omega} \cap V\left[G_{\gamma_{F, f}}\right]$ such that $f(x) \leq^{*} b_{F, f}$ for all $x \in F$. Let $\gamma(\alpha)$ be the supremum of all the $\gamma_{F, f}$ for $F, f$ as above. It is clear that the $\omega_{1}$-club $C \in V\left[G_{\omega_{2}}\right]$ of all $\alpha$ such that $\gamma(\beta)<\alpha$ for all $\beta<\alpha$ is as required.

Let $\mathrm{Q} \subset[X]^{\omega}$ be cofinal with respect to the inclusion. Fix a function $R$ : $\mathrm{Q} \rightarrow \mathcal{P}(X)$ assigning to each $Q \in \mathrm{Q}$ a $G_{\delta^{-}}$-subset $R(Q)$ of $\mathcal{P}(\omega)$ containing $Q$. By a standard argument (see, e.g., the proof of [6, Lemma 5.10]) there exists an $\omega_{1}$-club $D \subset \omega_{2}$ such that $\mathrm{Q} \cap V\left[G_{\alpha}\right] \in V\left[G_{\alpha}\right]$ and $R \uparrow(\mathrm{Q} \cap$ $\left.V\left[G_{\alpha}\right]\right) \in V\left[G_{\alpha}\right]$ for ${ }^{4}$ all $\alpha \in D$. Moreover, using $\mathrm{CH}$ in the intermediate models as in the previous paragraph, we may also assume that for every $Q_{0} \in\left[X \cap V\left[G_{\alpha}\right]\right]^{\omega} \cap V\left[G_{\alpha}\right]$ there exists $Q \in \mathrm{Q} \cap V\left[G_{\alpha}\right]$ such that $Q_{0} \subset Q$.

Let us fix $\alpha \in C \cap D$. We claim that $X \subset W$, where $W=\bigcup\{R(Q)$ : $\left.Q \in \mathrm{Q} \cap V\left[G_{\alpha}\right]\right\}$. Suppose that, contrary to our claim, there exists $p \in G_{\omega_{2}}$ and a $\mathbb{P}_{\omega_{2}}$-name $\dot{x}$ such that $p \Vdash \dot{x} \in \dot{X} \backslash \dot{W}$. By [15, Lemma 11] there is no loss of generality in assuming that $\alpha=0$. Applying [15, Lemma 14] to a sequence $\left\langle\dot{a}_{i}: i \in \omega\right\rangle$ such that $\dot{a}_{i}=\dot{x}$ for all $i \in \omega$, we get a condition $p^{\prime} \leq p$ such that $p^{\prime}(0) \leq{ }^{0} p(0)$, and a finite set $U_{s}$ of reals for every $s \in p^{\prime}(0)$ with $p^{\prime}(0)\langle 0\rangle \leq s$, such that for each $\varepsilon>0, s \in p^{\prime}(0)$ with $p^{\prime}(0)\langle 0\rangle \leq s$, and for all but finitely many immediate successors $t$ of $s$ in $p^{\prime}(0)$ we have

$$
p^{\prime}(0)_{t} \wedge p^{\prime} \uparrow\left[1, \omega_{2}\right) \Vdash \exists u \in U_{s}(|\dot{x}-u|<\varepsilon) .
$$

Fix $Q \in \mathrm{Q} \cap V$ containing $X \cap \bigcup\left\{U_{s}: s \in p^{\prime}(0), s \geq p^{\prime}(0)\langle 0\rangle\right\}$ and set $F=X \backslash R(Q)$. Note that $F$ is an $F_{\sigma}$-subset of $X$ coded in $V$. It follows that $p^{\prime} \Vdash \dot{x} \in \dot{F}$ because $p^{\prime}$ is stronger than $p$ that forces $\dot{x} \notin \dot{W} \supset \dot{X} \backslash \dot{F}$. Consider the map $f: F \rightarrow \omega^{S}$, where $S=\left\{s \in p^{\prime}(0): s \geq p^{\prime}(0)\langle 0\rangle\right\}$, defined as follows:

$$
f(y)(s)=\left[1 / \min \left\{|y-u|: u \in U_{s}\right\}\right]+1
$$

for $^{5}$ all $s \in S$ and $y \in F$. Since $F$ is disjoint from $Q$ which contains all the $U_{s}$ 's, $f$ is well defined. Since both $F$ and $f$ are coded in $V$, there exists $b \in \omega^{S} \cap V$ such that $f(y) \leq^{*} b$ for all $y \in F$.

It follows from $p^{\prime} \Vdash \dot{x} \in \dot{F}$ that $p^{\prime} \Vdash \dot{f}(\dot{x}) \leq^{*} b$, and hence there exists $p^{\prime \prime} \leq p$ and a finite subset $S_{0}$ of $S$ such that $p^{\prime \prime} \Vdash \dot{f}(\dot{x})(s) \leq b(s)$ for all $S \backslash S_{0}$. By replacing $p^{\prime \prime}$ with $p^{\prime \prime}(0)_{s}{ }^{\wedge} p^{\prime \prime} \uparrow\left[1, \omega_{2}\right)$ for some $s \in p^{\prime \prime}(0)$, if necessary, we may additionally assume that $p^{\prime \prime}(0)\langle 0\rangle \in S \backslash S_{0}$. Letting $s^{\prime \prime}=p^{\prime \prime}(0)\langle 0\rangle$, we conclude from the above that $p^{\prime \prime} \Vdash \dot{f}(\dot{x})\left(s^{\prime \prime}\right) \leq b\left(s^{\prime \prime}\right)$, which means that

$$
p^{\prime \prime} \Vdash \min \left\{|\dot{x}-u|: u \in U_{s^{\prime \prime}}\right\} \geq 1 / b\left(s^{\prime \prime}\right) .
$$

On the other hand, by our choice of $p^{\prime}$ and $p^{\prime \prime} \leq p^{\prime}$ we get that for all but finitely many immediate successors $t$ of $s^{\prime \prime}$ in $p^{\prime \prime}(0)$ we have

$$
p^{\prime \prime}(0)_{t}^{\wedge} p^{\prime \prime}\left\lceil\left[1, \omega_{2}\right) \Vdash \exists u \in U_{s^{\prime \prime}}|\dot{x}-u|<1 / b\left(s^{\prime \prime}\right)\right.
$$

which means $p^{\prime \prime}(0)_{t}{ }^{\prime \prime} p^{\prime \prime} \mid\left[1, \omega_{2}\right) \Vdash \min \left\{|\dot{x}-u|: u \in U_{s^{\prime \prime}}\right\}<1 / b\left(s^{\prime \prime}\right)$ and thus leads to a contradiction.

\footnotetext{
${ }^{4}$ Here by $R$ we mean the map which assigns to a $Q \in \mathrm{Q}$ some code of $\mathcal{P}(\omega) \backslash R(Q)$.

${ }^{5}$ Here $[a]$ is the largest integer not exceeding $a$.
} 
A subset $X$ of $\mathcal{P}(\omega)$ is called a $\lambda$-set if any $A \in[X]^{\omega}$ is a $G_{\delta^{-}}$-subset of $X$. Obviously, every weakly concentrated $\lambda$-set has size $\leq \omega_{1}$. Therefore Lemma 2.2 implies [19, Theorem 3.2] because the property of a subset of $\mathcal{P}(\omega)$ considered in the latter theorem easily implies being both Hurewicz and a $\lambda$-set, see, e.g., the proof of [31, Theorem 5] for details.

The next lemma can probably be considered as folklore. We present its proof for the sake of completeness.

Lemma 2.3. Let $Y \subset \mathcal{P}(\omega)$ be Hurewicz and $Q \subset \mathcal{P}(\omega)$ countable. Then for every $G_{\delta}$-subset $O$ of $\mathcal{P}(\omega)^{2}$ containing $Q \times Y$ there exists a $G_{\delta}$-subset $R \supset Q$ such that $R \times Y \subset O$.

Proof. Without loss of generality we shall assume that $O$ is open. Let us write $Q$ in the form $\left\{q_{n}: n \in \omega\right\}$ and set $O_{n}=\left\{z \in \mathcal{P}(\omega):\left\langle q_{n}, z\right\rangle \in\right.$ $O\} \supset Y$. For every $n$ find a cover $\mathcal{U}_{n}$ of $Y$ consisting of clopen subsets of $\mathcal{P}(\omega)$ contained in $O_{n}$. Let $\left\langle\mathcal{U}_{k}^{\prime}: k \in \omega\right\rangle$ be a sequence of open covers of $Y$ such that each $\mathcal{U}_{n}$ appears in it infinitely often. Applying the Hurewicz property of $Y$ we can find a sequence $\left\langle\mathcal{V}_{k}: k \in \omega\right\rangle$ such that $\mathcal{V}_{k} \in\left[\mathcal{U}_{k}\right]^{<\omega}$ and $Y \subset \bigcup_{k \in \omega} Z_{k}$, where $Z_{k}=\bigcap_{m>k} \cup \mathcal{V}_{m}$. Note that each $Z_{k}$ is compact and $Z_{k} \subset O_{n}$ for all $n \in \omega$ (because there exists $m \geq k$ such that $\mathcal{U}_{m}^{\prime}=\mathcal{U}_{n}$, and then $\left.Z_{k} \subset \cup \mathcal{V}_{m} \subset O_{n}\right)$. Thus $Q \times Y \subset Q \times\left(\bigcup_{k \in \omega} Z_{k}\right) \subset O$. Since $Z_{k}$ is compact, there exists for every $k$ an open $R_{k} \supset Q$ such that $R_{k} \times Z_{k} \subset O$. Set $R=\bigcap_{k \in \omega} R_{k}$ and note that $R \supset Q$ and $R \times Y \subset R \times \bigcup_{k \in \omega} Z_{k} \subset O$.

Let $A$ be a countable set and $x, y \in \omega^{A}$. As usually, $x \leq^{*} y$ means that $\{a \in A: x(a)>y(a)\}$ is finite. The smallest cardinality of an unbounded with respect to $\leq^{*}$ subset of $\omega^{\omega}$ is denoted by $\mathfrak{b}$. It is well known that $\omega_{1}<\mathfrak{b}$ in the Laver model, see [5] for this fact as well as systematic treatment of cardinal characteristics of reals.

The second part of Theorem 1.1 is a direct consequence of Lemma 2.2 and the following

Proposition 2.4. Suppose that $\mathfrak{b}>\omega_{1}$. Let $Y \subset \mathcal{P}(\omega)$ be a Hurewicz space and $X \subset \mathcal{P}(\omega)$ weakly concentrated. Then $X \times Y$ is Menger.

Proof. Fix a sequence $\left\langle\mathcal{U}_{n}: n \in \omega\right\rangle$ of covers of $X \times Y$ by clopen subsets of $\mathcal{P}(\omega)^{2}$. For every $Q \in[X]^{\omega}$ fix a sequence $\left\langle\mathcal{W}_{n}^{Q}: n \in \omega\right\rangle$ such that $\mathcal{W}_{n}^{Q} \in$ $\left[\mathcal{U}_{n}\right]^{<\omega}$ and $Q \times Y \subset \bigcap_{n \in \omega} \bigcup_{m \geq n} \cup \mathcal{W}_{m}^{Q}$. Letting $O_{Q}=\bigcap_{n \in \omega} \cup_{m \geq n} \cup \mathcal{W}_{m}^{Q}$ and using Lemma 2.3, we can find a $G_{\delta}$-subset $R_{Q} \supset Q$ such that $R_{Q} \times Y \subset O_{Q}$. Since $X$ is weakly concentrated, there exists $\mathrm{Q} \subset[X]^{\omega}$ of size $|\mathrm{Q}|=\omega_{1}$ such that $R=\bigcup\left\{R_{Q}: Q \in \mathrm{Q}\right\}$ contains $X$ as a subset. Let us fix $x \in X$ and find $Q \in \mathrm{Q}$ such that $x \in R_{Q}$. Then $\{x\} \times Y \subset R_{Q} \times Y \subset O_{Q}$. Therefore for every $\langle x, y\rangle \in X \times Y$ there exists $Q \in \mathrm{Q}$ such that $\langle x, y\rangle \in O_{Q}=$ $\bigcap_{n \in \omega} \bigcup_{m \geq n} \cup \mathcal{W}_{m}^{Q}$. Let us write $\mathcal{U}_{n}$ in the form $\left\{U_{k}^{n}: k \in \omega\right\}$ and for every $Q \in \mathrm{Q}$ fix a real $b_{Q} \in \omega^{\omega}$ with the property $\mathcal{W}_{n}^{Q} \subset\left\{U_{k}^{n}: k \leq b_{Q}(n)\right\}$. Since $|\mathrm{Q}|=\omega_{1}<\mathfrak{b}$, there exists $b \in \omega^{\omega}$ such that $b_{Q} \leq^{*} b$ for all $Q \in \mathrm{Q}$. It follows from the above that $X \times Y \subset \bigcup_{n \in \omega} \bigcup_{k \leq b(n)} U_{k}^{n}$, which completes our proof. 
A family $\mathcal{F} \subset[\omega]^{\omega}$ is called a semifilter if for every $F \in \mathcal{F}$ and $X \subset \omega$, if $|F \backslash X|<\omega$ then $X \in \mathcal{F}$.

The proof of the first part of Theorem 1.1 uses characterizations of the properties of Hurewicz and Menger obtained in [32]. Let $u=\left\langle U_{n}: n \in \omega\right\rangle$ be a sequence of subsets of a set $X$. For every $x \in X$ let $I_{s}(x, u, X)=\{n \in \omega$ : $\left.x \in U_{n}\right\}$. If every $I_{s}(x, u, X)$ is infinite (the collection of all such sequences $u$ will be denoted by $\left.\Lambda_{s}(X)\right)$, then we shall denote by $\mathcal{U}_{s}(u, X)$ the smallest semifilter on $\omega$ containing all $I_{s}(x, u, X)$. By [32, Theorem 3], a Lindelöf topological space $X$ is Menger (Hurewicz) if and only if for every $u \in \Lambda_{s}(X)$ consisting of open sets, the semifilter $\mathcal{U}_{s}(u, X)$ is Menger (Hurewicz). The proof given there also works if we consider only those $\left\langle U_{n}: n \in \omega\right\rangle \in \Lambda_{s}(X)$ such that all $U_{n}$ 's belong to a given base of $X$.

Proof of Theorem 1.1. Suppose that $X, Y$ are Hurewicz spaces such that $X \times Y$ is Lindelöf and fix $w=\left\langle U_{n} \times V_{n}: n \in \omega\right\rangle \in \Lambda_{s}(X \times Y)$ consisting of open sets. Set $u=\left\langle U_{n}: n \in \omega\right\rangle, v=\left\langle V_{n}: n \in \omega\right\rangle$, and note that $u \in \Lambda_{s}(X)$ and $v \in \Lambda_{s}(Y)$. It is easy to see that

$$
\mathcal{U}_{s}(w, X \times Y)=\left\{A \cap B: A \in \mathcal{U}_{s}(u, X), B \in \mathcal{U}_{s}(v, Y)\right\},
$$

and hence $\mathcal{U}_{s}(w, X \times Y)$ is a continuous image of $\mathcal{U}_{s}(u, X) \times \mathcal{U}_{s}(v, Y)$. By [32, Theorem 3] both of latter ones are Hurewicz, considered as subspaces of $\mathcal{P}(\omega)$, and hence their product is a Menger space by Proposition 2.4 and Lemma 2.2. Thus $\mathcal{U}_{s}(w, X \times Y)$ is Menger, being a continuous image of a Menger space. It now suffices to use [32, Theorem 3] again.

Acknowledgments. This research was supported by the Slovenian Research Agency grant P1-0292 and the Austrian Science Fund FWF grants I 1209-N25 and I 2374-N35. We would like to thank Marion Scheepers for fruitful discussions of products of Hurewicz spaces in various models of Borels's conjecture. We also thank Boaz Tsaban, as well as the referees, for many comments and suggestions which improved our presentation.

\section{REFERENCES}

[1] Aurichi, L.F., D-spaces, topological games, and selection principles, Topology Proc. 36 (2010), 107-122.

[2] Babinkostova, L., On some questions about selective separability, Math. Log. Quart. 55 (2009), 539-541.

[3] Barman, D.; Dow, A., Proper forcing axiom and selective separability, Topology Appl. 159 (2012), 806-813.

[4] Bartoszyński, T.; Judah, H., Set theory. On the structure of the real line. A. K. Peters, Ltd., Wellesley, MA, 1995.

[5] Blass, A., Combinatorial cardinal characteristics of the continuum, in: Handbook of Set Theory (M. Foreman, A. Kanamori, and M. Magidor, eds.), Springer, 2010, pp. 395-491.

[6] Blass, A.; Shelah, S., There may be simple $P_{\aleph_{1}}$ - and $P_{\aleph_{2}}$-points and the RudinKeisler ordering may be downward directed, Ann. Pure Appl. Logic 33 (1987), 213-243.

[7] Bukovsky, L., The structure of the real line. Instytut Matematyczny Polskiej Akademii Nauk. Monografie Matematyczne (New Series), 71. Birkhäuser/Springer Basel AG, Basel, 2011. 
[8] Chodounsky, D.; Guzman, O,; Hrušák, M., Mathias-Prikry and Laver type forcing; Summable ideals, coideals, and +-selective filters, Arch. Math. Logic 55 (2016), 493-504.

[9] Chodounský, D.; Repovš, D.; Zdomskyy, L., Mathias forcing and combinatorial covering properties of filters, J. Symb. Log. 80 (2015), 1398-1410.

[10] Gartside, P.; Medini, A.; Zdomskyy, L., The Tukey Order, Hyperspaces, and Selection Principles, work in progress.

[11] Hrušák, M.; van Mill, J., The existence of a meager in itself CDH metric space is independent of ZFC, Proc. Amer. Math. Soc., to appear.

[12] Hurewicz, W., Über die Verallgemeinerung des Borellschen Theorems, Math. Z. 24 (1925), 401-421.

[13] Hurewicz, W., Über Folgen stetiger Funktionen, Fund. Math. 9 (1927), 193204.

[14] Just, W.; Miller, A.W.; Scheepers, M.; Szeptycki, P.J., The combinatorics of open covers. II, Topology Appl. 73 (1996), 241-266.

[15] Laver, R., On the consistency of Borel's conjecture, Acta Math. 137 (1976), 151-169.

[16] Menger, K., Einige Überdeckungssätze der Punktmengenlehre, Sitzungsberichte. Abt. 2a, Mathematik, Astronomie, Physik, Meteorologie und Mechanik (Wiener Akademie) 133 (1924), 421-444.

[17] Miller, A., Rational perfect set forcing, in: Axiomatic Set Theory (J. Baumgartner, D. A. Martin, S. Shelah, eds.), Contemporary Mathematics 31, American Mathematical Society, Providence, Rhode Island, 1984, pp. 143-159.

[18] Miller, A. W., Special subsets of the real line, in: Handbook of Set-Theoretic Topology (K. Kunen, J.E. Vaughan, eds.), North Holland, Amsterdam, 1984, pp. 201-233.

[19] Miller, A.W.; Tsaban, B., Point-cofinite covers in the Laver model, Proc. Amer. Math. Soc. 138 (2010), 3313-3321.

[20] Miller, A.W.; Tsaban, B.; Zdomskyy, L., Selective covering properties of product spaces, Ann. Pure Appl. Logic 165 (2014), 1034-1057.

[21] Miller, A.W.; Tsaban, B.; Zdomskyy, L., Selective covering properties of product spaces, II: gamma spaces, Trans. Amer. Math. Soc. 368 (2016), 2865-2889.

[22] Repovš, D.; Zdomskyy, L., On M-separability of countable spaces and function spaces, Topology Appl. 157 (2010), 2538-2541.

[23] Sacks, G.E., Forcing with perfect closed sets, in: Axiomatic Set Theory (D. Scott eds.), Proc. Symp. in Pure Math. Vol. XIII, Part I. Am. Math. Soc., Providence, RI, 1971, 331-355.

[24] Scheepers, M., Combinatorics of open covers. I. Ramsey theory, Topology Appl. 69 (1996), 31-62.

[25] Scheepers, M.; Tall, F., Lindelöf indestructibility, topological games and selection principles, Fund. Math. 210 (2010), 1-46.

[26] Sierpiński, W., Sur un ensemble non dénombrable, dont toute image continue est de mesure nulle, Fund. Math. 11 (1928), 302-303.

[27] Scheepers, M.; Tsaban, B., The combinatorics of Borel covers, Topology Appl. 121 (2002), 357-382.

[28] Todorčević, S., Aronszajn orderings. Djuro Kurepa memorial volume, Publ. Inst. Math. (Beograd) (N.S.) 57(71) (1995), 29-46.

[29] Tsaban, B., Selection principles and special sets of reals, in: Open problems in topology II (edited By Elliott Pearl), Elsevier Sci. Publ., 2007, pp. 91-108.

[30] Tsaban, B., Algebra, selections, and additive Ramsey theory, preprint. http: //arxiv.org/pdf/1407.7437.pdf .

[31] Tsaban, B.; Zdomskyy, L., Hereditarily Hurewicz spaces and Arhangel'ski sheaf amalgamations, J. Eur. Math. Soc. (JEMS) 14 (2012), 353-372. 
[32] Zdomskyy, L., A semifilter approach to selection principles, Comment. Math. Univ. Carolin. 46 (2005), 525-539.

[33] Zdomskyy, L., Products of Menger spaces in the Miller model, work in progress.

Faculty of Education, and Faculty of Mathematics and Physics, University of Ljubljana \& Institute of Mathematics, Physics and Mechanics, 1000 Luubluana, Slovenia.

E-mail address: dusan.repovs@guest.arnes.si

Kurt Gödel Research Center for Mathematical Logic, University of Vienna, 1090 Vienna, Austria.

E-mail address: lzdomsky@gmail.com 